\title{
La peritoneale è una dialisi per "vecchi"?
}

\section{Michele Giannattasio ${ }^{1}$, Giuseppe Gernone ${ }^{2}$}

\author{
${ }^{1}$ Struttura Complessa di Nefrologia e Dialisi, Ospedale San Paolo, ASL Bari, Bari \\ ${ }^{2}$ Struttura Semplice Dipartimentale di Nefrologia e Dialisi, Ospedale Putignano, ASL Bari, Bari
}

\begin{abstract}
IS PERITONEAL DIALYSIS A METHOD FOR THE ELDERLY?
Abstract. An increasing number of elderly patients start dialysis. Despite peritoneal dialysis' potential benefits this technique is underutilized, particularly in the elderly. Recently, a debate entitled "Should peritoneal dialysis be the first option offered to the elderly who require renal re-placement therapy for continued longevity?" was published on Seminars in Dialysis.
\end{abstract}

Key words: Dialysis, Elderly, Shared decision-making

Conflict of interest: None.

Financial support: None.

Accettato: 19 Agosto 2013

La Dialisi Peritoneale (DP) è ormai una consolidata terapia dell'Uremia Cronica (UC), in quanto è considerata un'opzione terapeutica equivalente all'Emodialisi Periodica (HD) (1); è, inoltre, la tecnica dialitica domiciliare preferita. In Italia, circa il $10 \%$ della popolazione dialitica è in terapia sostitutiva peritoneale (2), mentre la popolazione dialitica globale in DP è pari a circa l'11\% (3).

La DP ha, quindi, una penetrazione bassa ed è rimasta una dialisi marginale negli ultimi lustri. Su questa rivista, 12 anni fa, ci ponemmo il problema di comprendere i motivi della mancata crescita della DP, malgrado fosse ormai indiscutibile la sua validità nell'ambito dei trattamenti sostitutivi della funzione renale.

Negli ultimi anni, invece, abbiamo assistito a una riduzione dei pazienti in DP e abbiamo cercato di capire perché ciò sia accaduto. Se ne è discusso anche nelle ultime quattro edizioni dei Minicorsi di Nefrologia a cura di colleghi esperti "peritonealisti", che hanno affrontato, interpretato il problema e proposto soluzioni (G. Cancarini: Quali motivi per la non crescita della DP in Italia, 2009; V. La Milia: Dialisi Peritoneale: quale futuro? 2010; G. Viglino: La Dialisi Peritoneale Assistita: ruolo in un programma di dialisi peritoneale, 2011; V. La Milia: Utilizzo attuale e possibilità di sviluppo della dialisi peritoneale in Italia, 2012).

I principali fattori condizionanti l'incidenza e la prevalenza della DP in Italia sono considerati (4):

- la presenza di Centri Privati, che non praticano DP

- l'esistenza di Centri Pubblici, che non praticano la DP (36\% dei Centri in Italia)

- l'esperienza dei Centri di Nefrologia nel praticare la DP, che consente un' adeguata prevalenza
- l'incremento dell'età anagrafica e dialitica dei pazienti

- la quota importante e in continuo incremento di pazienti molto anziani avviati alla dialisi.

Il Registro Americano (USRDS) riporta un incremento pari a ben il 50\% dei pazienti ultraottantenni in dialisi, confrontando i dati del 1996 con quelli del $2003(5,6)$. La dialisi nell'anziano è caratterizzata da un declino funzionale maggiore rispetto al paziente giovane; anche la qualità della vita nel paziente dializzato in HD o in DP è spesso aleatoria e imprevedibile (7).

Trattiamo, pertanto, una popolazione dialitica con un numero sempre crescente di comorbidità (8), per cui la sopravvivenza tende a ridursi nella coorte di dializzati anziani (5).

Nell'ambito della popolazione anziana (età superiore a 65 anni) la principale modalità di sostituzione della funzione renale è l'HD in-centro, mentre solo il $3.3 \%$ dei pazienti pratica la DP (6).

Pertanto, la DP è sottoutilizzata, specialmente nell'anziano, malgrado i potenziali vantaggi rispetto all'HD (ridotto stress emodinamico, buon controllo dell'anemia e minore uso di ESA, minore incidenza di aritmie cardiache, assenza di necessità di un accesso vascolare, mantenimento della funzione renale residua più a lungo rispetto all'HD, maggiore rimozione di $\beta_{2}$-Microglobulina e di Medie Molecole e trattamento dialitico a domicilio). I motivi clinici di tale sottoutilizzazione sono fondamentalmente legati a scarse capacità manuali, a ridotte funzioni neuro-psichiatriche, a una scarsa compliance, a ridotte capacità nutrizionali e a maggiori possibilità di infezione nell'anziano. Molto spesso, l'impedimento alla DP è legato alla necessità di avere un supporto assistenziale alle procedure dialitiche, che sempre 
più frequentemente non si trova nell'ambito della famiglia. La DP, quindi, potrà giocare un ruolo nel trattamento dell'UC solo se riusciremo a sviluppare la metodica in modo che la crescente popolazione dialitica, sempre più anziana e con sempre più comorbidità, possa avere l'opportunità di una terapia dialitica domiciliare, che, spesso, necessiterà di assistenza.

$\mathrm{Su}$ questa problematica è di recente comparso un dibattito sulla rivista Seminars in Dialysis tra due scuole di pensiero $(9,10)$, relativamente al fatto di considerare o meno la DP come terapia sostitutiva di prima scelta per il paziente anziano.

Nel dibattito è chiaramente evidenziato che la migliore terapia di sostituzione della funzione renale nel paziente anziano non è stata ancora individuata con certezza, per la mancanza di trial controllati e randomizzati che confrontino la DP con l'HD. I "non-peritonealisti”, come potremmo definire i nefrologi che non considerano la DP una prima scelta per la terapia sostitutiva dell'anziano, giustificano questo loro orientamento con la mancanza di differenze sostanziali nei risultati a lungo termine tra DP e HD in-centro, fatta eccezione per un leggero vantaggio della DP nei pazienti più giovani senza diabete mellito (DM), relativamente alla fase precoce di sostituzione della funzione renale. $\mathrm{La}$ DP, quindi, non sarebbe un'opzione vantaggiosa per quella grande coorte di popolazione in dialisi che, negli USA, è costituita dai pazienti anziani con DM.

È evidente che, in assenza di studi rigorosi, l'analisi della sopravvivenza per confrontare le due tecniche dialitiche è piena di insidie statistiche, né la scelta del trattamento può prescindere dalla stima della qualità della vita che, con uno specifico trattamento, possiamo assicurare al paziente, rapportando il tutto ai costi economici. È indubbio che la terapia dialitica fatta tra le mura domestiche sia la migliore opzione per il paziente anziano: si eviterebbero, così, non solo costosi trasferimenti dal e per il centro dialisi, che, a volte, necessitano di ambulanza, ma anche tutti quei disagi legati al trasferimento da effettuare anche con avverse condizioni climatiche o con temperature elevate.

I pazienti più anziani accettano generalmente il trattamento di dialisi, ma non lo scelgono, perché non autosufficienti; in genere, il principale interlocutore è un membro della famiglia che, spesso, sembra non essere in grado di ascoltare i consigli del nefrologo, in quanto impegnato a trovare la soluzione più rapida e meno impegnativa per sé e per la famiglia.

Il nefrologo deve fornire a paziente e/o familiari adeguate e corrette informazioni. L'approccio del medico, in alcuni casi, è quello di considerare la dialisi una necessità, che ha come unica alternativa la morte del paziente. Quasi mai vi è una discussione del "'futuro" del paziente, cioè cosa potrà succedere nei successivi mesi. L'indicazione a proseguire la terapia conservativa, da considerare a tutti gli effetti un'opzione terapeutica per l'anziano, è spesso valutata come un abbandono del paziente e della famiglia. Purtroppo, in alcune realtà, non è disponibile il supporto dello psicologo. Il nefrologo e lo staff nefrologico hanno il compito delicato di comunicare notizie "difficili" relative alla prognosi e alla terapia di pazienti anziani con scarse prospettive di vita. ̇̀ opportuno, quindi, che ci sia un adeguato background culturale in cure palliative; ciò migliora l'outcome e riduce i costi delle cure (ospedalizzazione e ricorso alla terapia intensiva) e, anche, l'ansia nel paziente e nella sua famiglia (11). Mai come nell'anziano dobbiamo porre indicazione alla terapia dialitica attraverso un processo decisionale condiviso solo se essa garantisce un reale miglioramento della qualità di vita del paziente.

\section{Riassunto}

Un numero sempre maggiore di pazienti anziani inizia la dialisi. Tuttavia, malgrado i potenziali vantaggi della DP rispetto all'HD, tale tecnica è sottoutilizzata, specialmente nell'anziano. È, di recente, comparso un dibattito sulla rivista Seminars in Dialysis, relativamente al fatto di considerare o meno la DP come terapia sostitutiva di prima scelta per il paziente anziano.

Parole chiave: Dialisi, Anziano, Processo decisionale condiviso

Dichiarazione di conflitto di interessi: Gli Autori dichiarano di non avere conflitto di interessi.

Contributi economici degli autori: Gli Autori dichiarano di non aver ricevuto sponsorizzazioni economiche per la preparazione dell'articolo.

Indirizzo degli Autori:

Dr. Michele Giannattasio

Struttura Complessa di Nefrologia e Dialisi

Ospedale San Paolo ASL Bari

Via Caposcardicchio nc

70123 Bari

michele.giannattasio@libero.it 


\section{Bibliografia}

1. Vonesh EF, Snyder JJ, Foley RN, Collins AJ. Mortality studies comparing peritoneal dialysis and hemodialysis: what do they tell us? Kidney Int Suppl 2006; (103): S3-11.

2. REGISTRO ITALIANO di DIALISI e TRAPIANTO: http:// www.sin-ridt.org/Italia/Report2009/ REPORT\% 20RIDT\%20 2009.pdf

3. Jain AK, Blake P, Cordy P, Garg AX. Global trends in rates of peritoneal dialysis. J Am Soc Nephrol 2012; 23 (3): 533-44.

4. Viglino G, Neri L, Alloatti S, et al. Analysis of the factors conditioning the diffusion of peritoneal dialysis in Italy. Nephrol Dial Transplant 2007; 22 (12): 3601-5.

5. Kurella M, Covinsky KE, Collins AJ, Chertow GM. Octogenarians and nonagenarians starting dialysis in the United States. Ann Intern Med 2007; 146 (3): 177-83.
6. USRD System: Excerpts from the USRDS 2005 annual data report: atlas of ESRD in the USA. Am J Kidney Dis 2006; 47 (Suppl. 1): S1; 2011 Atlas of CKD \& ESRD.

7. Cook WL, Jassal SV. Functional dependencies among the elderly on hemodialysis. Kidney Int 2008; 73: 1289-95.

8. Steinman TI. The Older Patient With End-Stage Renal Disease: Is Chronic Dialysis The Best Option? Semin Dial 2012; 25 (6): 602-5.

9. Bargman JM. Peritoneal dialysis should be the first choice for renal replacement therapy in the elderly. Semin Dial 2012; 25 (6): 668-70.

10. Mallappallil M, Patel A, Friedman EA. Peritoneal dialysis should not be the first choice for renal replacement therapy in the elderly. Semin Dial 2012; 25 (6): 671-4.

11. Singh P, Germain MJ, Cohen L, Unruh M. The elderly patient on dialysis: geriatric considerations. Nephrol Dial Transplant 2013. 